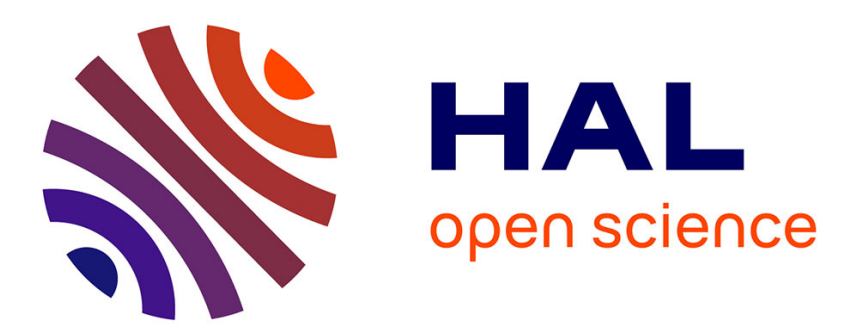

\title{
On Trefftz and weak Trefftz discontinuous Galerkin approaches for medium-frequency acoustics
}

\author{
Pierre Ladevèze, Hervé Riou
}

\section{To cite this version:}

Pierre Ladevèze, Hervé Riou. On Trefftz and weak Trefftz discontinuous Galerkin approaches for medium-frequency acoustics. Computer Methods in Applied Mechanics and Engineering, 2014, 278, pp.729-743. 10.1016/j.cma.2014.05.024 . hal-01647859

\section{HAL Id: hal-01647859 \\ https://hal.science/hal-01647859}

Submitted on 17 Dec 2019

HAL is a multi-disciplinary open access archive for the deposit and dissemination of scientific research documents, whether they are published or not. The documents may come from teaching and research institutions in France or abroad, or from public or private research centers.
L'archive ouverte pluridisciplinaire HAL, est destinée au dépôt et à la diffusion de documents scientifiques de niveau recherche, publiés ou non, émanant des établissements d'enseignement et de recherche français ou étrangers, des laboratoires publics ou privés. 


\title{
On Trefftz and weak Trefftz discontinuous Galerkin approaches for medium-frequency acoustics
}

\author{
Pierre Ladevèze*, Hervé Riou \\ LMT-Cachan (ENS Cachan/CNRS/Paris 6 University, PRES UniverSud Paris), 61 avenue du Pr'esident Wilson, F-94230 Cachan, France
}

\begin{abstract}
In this paper, the wave approach called the Variational Theory of Complex Rays (VTCR), which was developed for mediumfrequency acoustics and vibrations, is revisited as a discontinuous Galerkin method. Extensions leading to a weak Trefftz constraint are introduced. This weak Trefftz discontinuous Galerkin approach enables hybrid FEM/VTCR strategies to be developed easily, and paves the way for new computational techniques for the resolution of engineering problems. This paper presents some of the fundamental properties of the approach, which is illustrated by several numerical examples.
\end{abstract}

Keywords: Hybrid methods; Trefftz methods; Discontinuous Galerkin methods; VTCR; Acoustics

\section{Introduction}

In recent years, the use of numerical simulation techniques in the design, analysis and optimization of systems has become an indispensable part of the industrial design process. The standard Galerkin Finite Element Method (FEM) [1] is a well-established computer-aided engineering tool commonly used for the analysis of time-harmonic dynamic problems. However, using continuous, piecewise polynomial shape functions leads to very large numerical models and, in practice, restricts applications of this prediction technique to the low-frequency range.

Trefftz methods [2] have been proposed as a means to overcome this limitation. They differ from the FEM in the shape functions they use for the expansion of the field variables, which are exact solutions of the governing differential equations. Compared to finite element methods, these functions often lead to a considerable reduction in model size and computational effort. Some examples of such methods are: a special version of the partition of unity method [3], the ultra weak variational method [4,5], the plane wave discontinuous Galerkin method [6,7], the least-squares method [8,9], the discontinuous enrichment method [10,11], the element-free Galerkin method [12], the wave boundary element method $[13,14]$ and the wave-based method $[15,16]$. Some mathematical results regarding the convergence of these methods can be found in [6,7]. The Variational Theory of Complex Rays (VTCR), first

\footnotetext{
* Corresponding author.

E-mail addresses: pierre.ladeveze@1mt.ens-cachan.fr, herve92160@gmail.com (P. Ladevèze), herve.riou@1mt.ens-cachan.fr (H. Riou).
} 


\section{Nomenclature}

\begin{tabular}{|c|c|}
\hline$\Omega$ & Acoustic domain \\
\hline$\partial \Omega$ & Boundary of $\Omega$ \\
\hline$u$ or $v$ & Acoustic pressure \\
\hline & Acoustic wave number \\
\hline$\eta$ & Damping coefficient \\
\hline$h$ & Constant related to the acoustic impedance \\
\hline$r_{d}$ & Acoustic source prescribed over $\Omega$ \\
\hline$g_{d}$ & Acoustic source prescribed over $\partial_{2} \Omega$ \\
\hline$u_{d}$ & Acoustic pressure prescribed over $\partial_{1} \Omega$ \\
\hline $\mathbf{q}_{u}$ & Pressure flux proportional to $\operatorname{grad} u$ \\
\hline$\Omega_{E}$ & Subdomain of $\Omega$ \\
\hline$\Gamma_{E E^{\prime}}$ & Interface between subdomains $\Omega_{E}$ and $\Omega_{E}^{\prime}$ \\
\hline
\end{tabular}

introduced in [17] for steady-state vibration problems and in [18] for transient problems, also belongs to that category. The main differences in these methods lie essentially in the treatment of the transmission conditions at the boundaries of the elements or substructures.

The main characteristic of the VTCR, the method discussed in this paper, is the use of a specific weak formulation of the problem which enables the approximations within the substructures to be a priori independent of one another. Thus, any type of shape function can be used within a given substructure provided it satisfies the governing equation, thus giving the approach great flexibility.

As explained in $[17,19,20]$, the VTCR was originally developed as an extension to acoustics and vibrations of the formulation introduced in [21]. However, since the shape functions are discontinuous, there is a link between the VTCR and the discontinuous Galerkin methods studied in this paper. Discontinuous Galerkin methods represent a vast domain (see [22] or [23] for an overview, unified analysis and comparisons). Our discontinuous Galerkin formulation, which is nonsymmetrical, can be viewed as the Trefftz version of Baumann-Oden's discontinuous Galerkin formulation [24,25]. The main engineering applications addressed are car acoustics [26] and pyrotechnic shock propagation in space launchers [18].

In addition, this paper introduces extensions of the classical VTCR formulation in which the Trefftz constraint is weakened. This leads to a new numerical method which can be called the weak Trefftz discontinuous Galerkin method. These extensions allow for an easy coupling of different types of numerical models, including classical finite element models. As a consequence, they lead to new approaches to the resolution of engineering problems. Another application concerns structures which are not piecewise homogeneous. In this paper, some fundamental properties of this new method will be presented and illustrated by numerical examples. Our standard problem is the 2D or 3D acoustic problem.

First, in Section 2, the VTCR approach is reviewed. Then, in Section 3, the weak Trefftz discontinuous Galerkin formulation is introduced as a discontinuous Galerkin method and two examples of finite element approaches are examined in detail. In Section 4, hybrid FEM/VTCR approaches are introduced as special cases of the weak Trefftz discontinuous Galerkin formulation. Section 5 presents several applications which illustrate the proposed computational technique for medium-frequency vibration problems.

\section{The Variational Theory of Complex Rays: a Trefftz discontinuous Galerkin formulation}

\subsection{The reference problem}

Our reference problem is a standard acoustic problem defined over domain $\Omega$ with boundaries $\partial \Omega=\partial_{1} \Omega \cup \partial_{2} \Omega$ (see Fig. 1 on the left): find $u \in H^{1}(\Omega)$ such that

$$
\left\{\begin{array}{l}
(1+i \eta) \Delta u+k^{2} u+r_{d}=0 \quad \text { over } \Omega \\
u=u_{d} \text { over } \partial_{1} \Omega \\
(1+i \eta) \partial_{\mathbf{n}} u+h i k u=g_{d} \quad \text { over } \partial_{2} \Omega
\end{array}\right.
$$



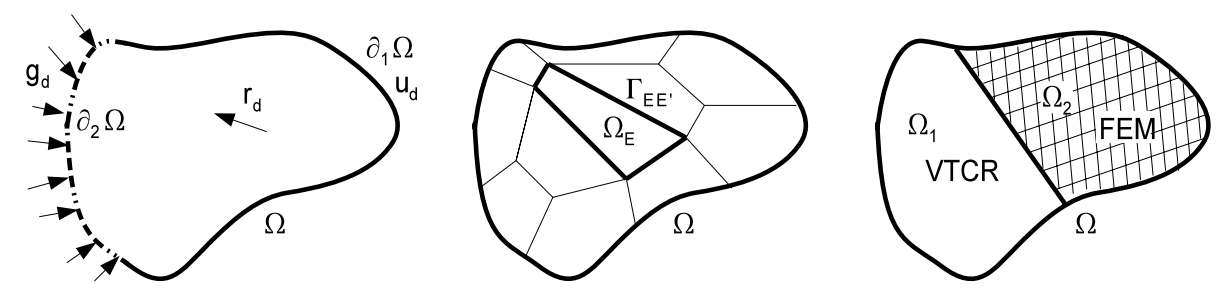

Fig. 1. Left: definition of the computational domain $\Omega$. Middle: definition of the subdomains of $\Omega$. Right: coupling of the FEM and the VTCR descriptions (see Section 4).

where $\partial_{\mathbf{n}} u=\operatorname{grad} u \cdot \mathbf{n}, \mathbf{n}$ being the outward normal; $u$ is the acoustic pressure; $k$ is the wave number, which is proportional to the frequency of the problem; $h$ is a constant related to the acoustic impedance; $r_{d}$ and $g_{d}$ are prescribed acoustic sources over $\Omega$ and $\partial_{2} \Omega$, and $u_{d}$ is the prescribed acoustic pressure over $\partial_{1} \Omega$. The damping coefficient $\eta$ is positive or equal to zero. $k$ and $h$ are real, positive and constant values. The prescribed data are $r_{d}, u_{d}$ and $g_{d}$, which are assumed to be regular enough for a unique solution to exist in $H^{1}(\Omega)$ if $\eta>0$ (or if $\eta=0$, but the area in 3D or the length in $2 \mathrm{D}$ of $\partial_{2} \Omega$ is nonzero). The first equation of (1) corresponds to the classical Helmholtz acoustic problem. The two boundary conditions correspond to a pressure or to an impedance prescribed over $\partial \Omega$.

\subsection{The variational formulation of the reference problem associated with the VTCR}

Let us first introduce an equivalent formulation of Problem (1) if its solution belongs to $\mathcal{U} \subset H^{1}(\Omega)$. Domain $\Omega$ is divided into subdomains $\Omega_{E}$, with $E \in \mathbf{E}$ (see the middle of Fig. 1). The interface between two subdomains $E$ and $E^{\prime}$ is denoted $\Gamma_{E E^{\prime}}$. The interface between $\Omega_{E}$ and boundary $\partial \Omega$ (when it exists) is denoted $\Gamma_{E E}$. The VTCR is a Trefftz approach which uses the affine space:

$$
\mathcal{U}=\left\{u \mid u_{\mid \Omega_{E}} \in \mathcal{U}_{E}\right\}
$$

with

$$
\mathcal{U}_{E}=\left\{u_{E} \mid u_{E} \in \mathcal{V}_{E} \subset H^{1}\left(\Omega_{E}\right) ;(1+i \eta) \Delta u_{E}+k^{2} u_{E}+r_{d}=0 \text { on } \Omega_{E}\right\} .
$$

The vector spaces (with $r_{d}=0$ ) associated with $\mathcal{U}$ and $\mathcal{U}_{E}$ are denoted $\mathcal{U}_{0}$ and $\mathcal{U}_{E, 0}$.

Now, in order to develop the discontinuous Galerkin approach, let us denote classically:

$$
\begin{aligned}
& \{u\}_{E E^{\prime}}=\left(u_{E}+u_{E^{\prime}}\right)_{\mid \Gamma_{E E^{\prime}}} \\
& {[u]_{E E^{\prime}}=\left(u_{E}-u_{E^{\prime}}\right)_{\mid \Gamma_{E E^{\prime}}} .}
\end{aligned}
$$

Denoting $\mathbf{q}_{\mathbf{u}}=(1+i \eta) \operatorname{grad} u$, the VTCR formulation can be written: find $u \in \mathcal{U}$ such that

$$
\begin{aligned}
& \operatorname{Re}\left(-i k\left(\sum_{E, E^{\prime} \in \mathbf{E}} \int_{\Gamma_{E E^{\prime}}}\left(\frac{1}{2}\left\{\mathbf{q}_{\mathbf{u}} \cdot \mathbf{n}\right\}_{E E^{\prime}}\{\tilde{v}\}_{E E^{\prime}}-\frac{1}{2}\left[\tilde{\mathbf{q}}_{\mathbf{v}} \cdot \mathbf{n}\right]_{E E^{\prime}}[u]_{E E^{\prime}}\right) d S\right.\right. \\
& \quad-\sum_{E \in \mathbf{E}} \int_{\Gamma_{E E \cap \partial_{1} \Omega} \tilde{\mathbf{q}}_{\mathbf{v}} \cdot \mathbf{n}\left(u-u_{d}\right) d S} \\
& \left.\left.\quad+\sum_{E \in \mathbf{E}} \int_{\Gamma_{E E} \cap \partial_{2} \Omega} \frac{1}{2}\left(-\tilde{\mathbf{q}}_{\mathbf{v}} \cdot \mathbf{n}\left(u+\left(\mathbf{q}_{\mathbf{u}} \cdot \mathbf{n}-g_{d}\right) /(h i k)\right)+\tilde{v}\left(\mathbf{q}_{\mathbf{u}} \cdot \mathbf{n}+h i k u-g_{d}\right)\right) d S\right)\right) \\
& =0 \quad \forall v \in \mathcal{U}_{0}
\end{aligned}
$$

where $\tilde{\square}$ and $\operatorname{Re}(\square)$ represent respectively the complex conjugate part and the real part of a quantity $\square$. The quantity involved in (5) is a dissipation. It is clear that (5) is satisfied by the exact solution of (1). All that has to be done to get an approximation is to replace $\mathcal{U}_{E}$ by the finite-dimension subspace $\mathcal{U}_{E}^{h}$. The associated spaces are $\mathcal{U}^{h}$ and $\mathcal{U}_{0}^{h}$. It can also be noted that it is possible to suppress the real part in (5) because the resulting problem is equivalent to (5). This will be done in the next sections to generate analytical results. 


\subsection{Properties of the VTCR formulation}

First, let us note that Formulation (5) can be written: find $u \in \mathcal{U}$ such that

$$
b(u, v)=l(v) \quad \forall v \in \mathcal{U}_{0}
$$

where $b$ is $u$-linear in $\mathcal{U}$ and $v$-(anti)linear in $\mathcal{U}_{0}$, and $l$ is $v$-(anti)linear in $\mathcal{U}_{0}$. In addition, $b$ is such that $b(u, u)$ is real.

Let us introduce

$$
\|u\|_{\mathcal{U}}^{2}=\sum_{E \in \mathbf{E}} \int_{\Omega_{E}} \operatorname{grad} u \cdot \operatorname{grad} \tilde{u} d \Omega .
$$

Property 1. $\|u\|_{\mathcal{U}}$ is a norm over $\mathcal{U}_{0}$.

Proof. The only condition which is not straightforward is $\|u\|_{\mathcal{U}}=0$ for $u \in \mathcal{U}_{0} \Rightarrow u=0$ over $\Omega$. Assuming that $u \in \mathcal{U}_{0}$ such that $\|u\|_{\mathcal{U}}=0$, it follows that $\mathbf{q}_{\mathbf{u}}=\mathbf{0}$ over $\Omega$. Hence, from $\operatorname{div} \mathbf{q}_{\mathbf{u}}+k^{2} u=0$ over $\Omega_{E}$ with $E \in \mathbf{E}$, we have $u=0$ over $\Omega_{E}$ and, consequently, over $\Omega$.

Property 2. For $u \in \mathcal{U}_{0}, b(u, u) \geq k \eta\|u\|_{\mathcal{U}}^{2}$, which means that if $\eta$ is positive the formulation is coercive.

Proof. For $u \in \mathcal{U}_{0}$, we have

$$
b(u, u)=\operatorname{Re}\left(-i k\left(\sum_{E \in \mathbf{E}} \int_{\partial \Omega_{E}} \mathbf{q u} \cdot \mathbf{n} \tilde{u} d S+\sum_{E \in \mathbf{E}} \frac{1}{2} \int_{\partial \Omega_{E} \cap \partial_{2} \Omega}\left(-\left(\mathbf{q}_{\mathbf{u}} \cdot \mathbf{n}\right)\left(\tilde{\mathbf{q}}_{\mathbf{u}} \cdot \mathbf{n}\right) /(h i k)+h i k u \tilde{u}\right) d S\right)\right) .
$$

Consequently,

$$
\begin{aligned}
b(u, u)= & \operatorname{Re}\left(-i k \sum_{E \in \mathbf{E}} \int_{\Omega_{E}}\left(-k^{2} u \tilde{u}+(1+i \eta) \operatorname{grad} u \cdot \operatorname{grad} \tilde{u}\right) d \Omega\right) \\
& +\sum_{E \in \mathbf{E}} \frac{1}{2} \int_{\partial \Omega_{E} \cap \partial_{2} \Omega}\left(\left(\mathbf{q}_{\mathbf{u}} \cdot \mathbf{n}\right)\left(\tilde{\mathbf{q}}_{\mathbf{u}} \cdot \mathbf{n}\right) / h+h k^{2} u \tilde{u}\right) d S .
\end{aligned}
$$

Finally,

$$
b(u, u)=k \eta \sum_{E \in \mathbf{E}} \int_{\Omega_{E}} \operatorname{grad} u \cdot \operatorname{grad} \tilde{u} d \Omega+\sum_{E \in \mathbf{E}} \frac{1}{2} \int_{\partial \Omega_{E} \cap \partial_{2} \Omega}\left(\left(\mathbf{q}_{\mathbf{u}} \cdot \mathbf{n}\right)\left(\tilde{\mathbf{q}}_{\mathbf{u}} \cdot \mathbf{n}\right) / h+h k^{2} u \tilde{u}\right) d S .
$$

Then, $b(u, u) \geq k \eta\|u\|_{\mathcal{U}}^{2}$.

Property 1 implies that if $\eta$ is positive the solution of (5) is unique. Since the exact solution of Problem (1) verifies (5), Formulation (5) is equivalent to the reference problem (1). Besides, it can be observed that for a perturbation $\Delta l \in \mathcal{U}_{0}^{\prime}$ of the excitation the perturbation $\Delta w$ of the solution verifies

$$
\|\Delta w\|_{\mathcal{U}} \leq \frac{1}{k \eta}|\Delta l|_{\mathcal{U}_{0}^{\prime}} .
$$

Moreover, all the properties demonstrated above remain valid if one replaces $\mathcal{U}_{0}$ by $\mathcal{U}_{0}^{h}$. Thus, seeking a numerical approximation makes sense. An illustration of the use of (5) can be found in [27-29].

\section{The weak Trefftz discontinuous Galerkin formulation}

In the VTCR, the governing equation is satisfied within each subdomain $\Omega_{E}, E \in \mathbf{E}$ (see Section 2). The objective of this section is to weaken this condition in order to enable, for example, the use of the FEM solutions. Let $\mathcal{U}^{h}$ and 
$\mathcal{U}_{0}^{h}$ denote respectively the working space and the associated vector space, examples of which will be given later. The weak Trefftz discontinuous Galerkin formulation consists in finding $u \in \mathcal{U}^{h}$ such that

$$
\begin{aligned}
& \operatorname{Re}\left(-i k\left(\sum_{E, E^{\prime} \in \mathbf{E}} \int_{\Gamma_{E E^{\prime}}}\left(\frac{1}{2}\left\{\mathbf{q}_{\mathbf{u}} \cdot \mathbf{n}\right\}_{E E^{\prime}}\{\tilde{v}\}_{E E^{\prime}}-\frac{1}{2}\left[\tilde{\mathbf{q}}_{\mathbf{v}} \cdot \mathbf{n}\right]_{E E^{\prime}}[u]_{E E^{\prime}}\right) d S\right.\right. \\
& \quad-\sum_{E \in \mathbf{E}} \int_{\Gamma_{E E} \cap \partial_{1} \Omega} \tilde{\mathbf{q}}_{\mathbf{v}} \cdot \mathbf{n}\left(u-u_{d}\right) d S \\
& \quad+\sum_{E \in \mathbf{E}} \int_{\Gamma_{E E} \cap \partial_{2} \Omega} \frac{1}{2}\left(-\tilde{\mathbf{q}}_{\mathbf{v}} \cdot \mathbf{n}\left(u+\left(\mathbf{q}_{\mathbf{u}} \cdot \mathbf{n}-g_{d}\right) /(h i k)\right)+\tilde{v}\left(\mathbf{q}_{\mathbf{u}} \cdot \mathbf{n}+i h k u-g_{d}\right)\right) d S \\
& \left.\left.\quad-\sum_{E \in \mathbf{E}} \frac{1}{2} \int_{\Omega_{E}}\left(\left(\operatorname{div} \mathbf{q}_{\mathbf{u}}+k^{2} u+r_{d}\right) \tilde{v}-\left(\operatorname{div} \tilde{\mathbf{q}}_{\mathbf{v}}+k^{2} \tilde{v}\right) u\right) d \Omega\right)\right)=0 \quad \forall v \in \mathcal{U}_{0}^{h} .
\end{aligned}
$$

Again, the real part in (12) can be omitted if desired. This will be done in the next sections to generate analytical results. Let us note that another similar formulation exists if the last integral in (12) is replaced by $-\sum_{E \in \mathbf{E}}$ $\int_{\Omega_{E}}\left(\operatorname{div} \mathbf{q u}_{\mathbf{u}}+k^{2} u+r_{d}\right) \tilde{v} d \Omega$.

\subsection{Properties of the weak Trefftz discontinuous Galerkin formulation}

Let us note that (12) can be written as: find $u \in \mathcal{U}^{h}$ such that

$$
b(u, v)=l(v) \quad \forall v \in \mathcal{U}_{0}^{h}
$$

where $b$ and $l$, which are $u$-linear in $\mathcal{U}$, are also $v$-(anti)linear in $\mathcal{U}_{0}$. In addition, $b$ is such that $b(u, u)$ is real. If $\mathcal{U}^{h}$ and $\mathcal{U}_{0}^{h}$ are the spaces used in the VTCR, then the local interior equation is satisfied and we arrive back at Formulation (5) or Formulation (6).

Property 3. For $u \in \mathcal{U}_{0}$, we have

$$
b(u, u)=\sum_{E \in \mathbf{E}} k \eta \int_{\Omega_{E}} \operatorname{grad} \tilde{u} \cdot \operatorname{grad} u d \Omega+\sum_{E \in \mathbf{E}} \int_{\Gamma_{E E} \cap \partial_{2} \Omega} \frac{1}{2}\left(\left(\mathbf{q}_{\mathbf{u}} \cdot \mathbf{n}\right)\left(\tilde{\mathbf{q}}_{\mathbf{u}} \cdot \mathbf{n}\right) / h+h k^{2} u \tilde{u}\right) d S \geq 0 .
$$

Proof.

$$
\begin{aligned}
b(u, u)= & \operatorname{Re}\left(-i k\left(\sum_{E \in \mathbf{E}} \int_{\partial \Omega_{E}}\left(\mathbf{q}_{\mathbf{u}} \cdot \mathbf{n}\right) \tilde{u} d S-\sum_{E \in \mathbf{E}} \int_{\Omega_{E}} \operatorname{div} \mathbf{q}_{\mathbf{u}} \tilde{u} d \Omega\right.\right. \\
& \left.\left.\times \sum_{E \in \mathbf{E}} \int_{\Gamma_{E E} \cap \partial_{2} \Omega} \frac{1}{2}\left(\left(\mathbf{q}_{\mathbf{u}} \cdot \mathbf{n}\right)\left(\tilde{\mathbf{q}}_{\mathbf{u}} \cdot \mathbf{n}\right) / h+h k^{2} u \tilde{u}\right) d S\right)\right) .
\end{aligned}
$$

Consequently,

$$
\begin{aligned}
b(u, u)= & k \eta \sum_{E \in \mathbf{E}} \int_{\Omega_{E}} \operatorname{grad} u \cdot \operatorname{grad} \tilde{u} d \Omega \\
& +\sum_{E \in \mathbf{E}} \int_{\Gamma_{E E} \cap \partial_{2} \Omega} \frac{1}{2}\left(\left(\mathbf{q}_{\mathbf{u}} \cdot \mathbf{n}\right)\left(\tilde{\mathbf{q}}_{\mathbf{u}} \cdot \mathbf{n}\right) / h+h k^{2} u \tilde{u}\right) d S .
\end{aligned}
$$

It can be observed that if $b(u, u)=0$, then $u$ is piecewise constant within subdomains $\Omega_{E}, E \in \mathbf{E}$ and is not equal to zero. Consequently, the shape functions belonging to $\mathcal{U}_{0}^{h}$ should satisfy the following condition (P):

Condition (P) Let $a_{E} \in \mathcal{U}_{E}^{h}$ be a piecewise constant function within subdomains $E \in$ E. $a_{E}$ satisfies condition (P) if

$$
\left\{\forall v \in \mathcal{U}_{0}^{h}, \forall E \in \mathbf{E}, \operatorname{Re}\left(-i k\left(\sum_{E, E^{\prime} \in \mathbf{E}} \int_{\partial \Omega_{E}}\left(\mathbf{q}_{\mathbf{v}} \cdot \mathbf{n}\right) \tilde{a}_{E^{\prime}} d S\right)\right)=0\right\} \Rightarrow a_{E}= \pm a
$$


where $E^{\prime}$ is a subdomain sharing a common boundary with $E$, with the convention $a_{E^{\prime}}=-a_{E}$ over $\partial \Omega_{E} \cap \partial \Omega$. It will be shown below that this condition is not restrictive and that it enables the following uniqueness property of the approximate problem to be proved.

Property 4. If $\mathcal{U}_{0}^{h}$ satisfies condition (P) and if $\eta$ is positive, the weak Trefftz discontinuous Galerkin formulation (12) has a unique solution.

Proof. Let us consider two solutions $u^{1}$ and $u^{2}$ of (12). $v=u^{1}-u^{2} \in \mathcal{U}_{0}^{h}$ and

$$
b(v, v)=\sum_{E \in \mathbf{E}} k \eta \int_{\Omega_{E}} \operatorname{grad} v \cdot \operatorname{grad} \tilde{v} d \Omega+\sum_{E \in \mathbf{E}} \int_{\Gamma_{E E} \cap \partial_{2} \Omega} \frac{1}{2}\left(\left(\mathbf{q}_{\mathbf{v}} \cdot \mathbf{n}\right)\left(\tilde{\mathbf{q}}_{\mathbf{v}} \cdot \mathbf{n}\right) / h+h k^{2} v \tilde{v}\right) d S=0 .
$$

It follows that $v_{E}=a_{E}$ with $E \in \mathbf{E}$, where $a_{E}$ is piecewise constant within the subdomains and $a_{E}=0$ in the subdomains sharing a common boundary with $\partial_{2} \Omega$. Back-substituting this results into (12), one also finds $b\left(v, v^{*}\right)=0 \forall v^{*} \in \mathcal{U}_{0}^{h}$, which leads to

$$
\forall v^{*} \in \mathcal{U}_{0}^{h}, \quad \operatorname{Re}\left(-i k\left(\sum_{E, E^{\prime} \in \mathbf{E}} \int_{\partial \Omega_{E}}\left(\mathbf{q}_{\mathbf{v}}^{*} \cdot \mathbf{n}\right) \tilde{a}_{E^{\prime}} d S\right)\right)=0
$$

where $E^{\prime}$ denotes the subdomains sharing a common boundary with $E$, with the convention $a_{E^{\prime}}=-a_{E}$ over $\partial \Omega_{E} \cap \partial \Omega$. (19) can be identified with condition (P). Consequently, $a_{E}= \pm a \forall E \in \mathbf{E}$. Moreover, given that $a_{E}=0$ over $\partial_{2} \Omega$, then $a=0$.

\subsection{First example of the use of the weak Trefftz discontinuous Galerkin formulation}

Here, we consider that the FE method is used for each subdomain $E \in \mathbf{E}$. If desired, the meshes and element types can be completely different across subdomains. For a subdomain $E$, we introduce the finite element subspace $\mathcal{V}_{E}^{h}$, and for $u^{h} \in \mathcal{V}_{E}^{h}$ we prescribe

$$
\int_{\Omega_{E}}\left(k^{2} u^{h}+\operatorname{div} \mathbf{q}_{\mathbf{u}}^{\mathbf{h}}+r_{d}\right) \tilde{u}^{*} d \Omega=0 \quad \forall u^{*} \in \mathcal{V}_{E, 0}^{h}=\left\{u \mid u \in \mathcal{V}_{E}^{h}, u_{\mid \partial \Omega_{E}}=0\right\} .
$$

The corresponding subspace is $\mathcal{U}_{E}^{h}$.

Property 5. If $\mathcal{U}_{E}^{h}$ satisfies (20), if the number of internal modes is nonzero and if $\eta$ is positive, then

- condition (P) holds

- $\|\square\|=(b(\square, \square))^{1 / 2}$ is a norm over $\mathcal{U}_{0}^{h}$

- the weak Trefftz discontinuous Galerkin formulation has a unique solution.

Proof. If $u^{h} \in \mathcal{U}_{E, 0}^{h}$, then $\int_{\Omega_{E}}\left(k^{2} u^{h}+\operatorname{div} \mathbf{q}_{\mathbf{u}^{\mathbf{h}}}\right) \tilde{u}^{*} d \Omega=0 \forall u^{*} \in \mathcal{V}_{E, 0}^{h}$. Then, for $u^{h}$ constant over $\Omega_{E}$, we find that $u_{h} \int_{\Omega_{E}} k^{2} \tilde{u}^{*} d \Omega=0 \forall u^{*} \in \mathcal{V}_{E, 0}^{h} \forall E \in \mathbf{E}$. Thus, if the number of internal finite element nodes is nonzero for each subdomain $E \in \mathbf{E}$, then $u^{h}=0$ throughout $\Omega$. The last two points of Property 5 are direct consequences of the proofs above.

\subsection{Second example of the use of the weak Trefftz discontinuous Galerkin formulation}

Now, we consider that each subdomain consists of a single element. These elements can be completely different. The weak Trefftz discontinuous Galerkin formulation (12) remains unchanged. Some constraints can be added in order to satisfy the interior equation in an average sense, but these conditions are unnecessary in our case. However, condition $(\mathrm{P})$ must be satisfied.

Property 6. Condition (P) is always satisfied for any finite element of order $n$ (hereafter denoted $P_{n}$-element). 
Proof. Let us rewrite condition (P). $a_{E} \in \mathcal{U}_{E}^{h}, E \in \mathbf{E}$ is a piecewise constant field. We should find $\operatorname{Re}\left(-i k\left(\sum_{E, E^{\prime} \in \mathbf{E}}\right.\right.$ $\left.\left.\int_{\partial \Omega_{E}}\left(\mathbf{q}_{\mathbf{v}} \cdot \mathbf{n}\right) \tilde{a}_{E^{\prime}} d S\right)\right)=0 \Rightarrow a_{E}= \pm a \forall E \in \mathbf{E}$ with $a_{E}=-a_{E}$ on $\partial \Omega_{E} \cap \partial \Omega$. Let us focus on $P_{1}$-elements. If condition (P) is satisfied for $P_{1}$-elements, obviously it is also satisfied for $P_{n}$-elements. For $P_{1}$-elements, one has $\int_{\partial \Omega_{E}} a_{E^{\prime}} \mathbf{n} d S$; then, $\forall E \in \mathbf{E} a_{E^{\prime}}=\alpha_{E}\left(\alpha_{E}\right.$ constant over $\left.\Omega_{E}\right)$ for any subdomain $E^{\prime}$ sharing a common boundary with $E$.

Let us introduce $z_{E}=\alpha_{E}+a_{E} \cdot z_{E}$ is continuous because $z_{E \mid \Gamma_{E E^{\prime}}}=a_{E^{\prime}}+a_{E}=z_{E^{\prime} \mid \Gamma_{E E^{\prime}}}$. It follows that $z$ is constant over $\Omega$. Since $z$ is zero over $\partial \Omega, z=0$ over $\Omega$ and $\alpha_{E}=-a_{E}$. Consequently, $a_{E}$ can take only the values $+a$ or $-a, a$ being a constant over $\Omega$.

Property 7. In the case where each subdomain is associated with an independent $P_{n}$-element approximation, the weak Trefftz discontinuous Galerkin formulation (12) has a unique solution.

Proof. The result is a consequence of Properties 4 and 6.

To conclude this discussion, it is important to introduce a norm over $\mathcal{U}_{0}^{h}$. Let us observe that, here, $(b(\square, \square))^{1 / 2}$ is not a norm (see the previous properties); therefore, it is necessary to build a new norm. In order to do that, let us introduce the space

$$
\overline{\mathcal{U}}_{E, 0}^{h}=\left\{u \mid u \in \mathcal{V}_{E}^{h}, u=\mathbf{C}_{\mathbf{E}} \cdot \mathbf{X}_{\mathbf{E}}\right\}
$$

where $\mathbf{C}_{\mathbf{E}}$ is constant over $\Omega_{E}$ and $\mathbf{X}_{\mathbf{E}}$ is the position vector relative to the center of inertia of element $E . \overline{\mathcal{U}}_{E, 0}^{h}$ is a subspace of the space related to the $P_{1}$-element. The associated space defined over $\Omega$ is denoted $\overline{\mathcal{U}}_{0}^{h}$. Now, let us introduce, for $u \in \mathcal{U}_{0}^{h}$, the quantity:

$$
\gamma(u)=\sup _{v \in \overline{\mathcal{U}}_{0}^{h}} b(u, v) /\left\|\mathbf{C}_{\mathbf{v}}\right\|_{L^{2}(\Omega)}
$$

where $\mathbf{C}_{\mathbf{v}}$ corresponds to the vector $\mathbf{C}_{\mathbf{E}}$ of $v$ according to (21).

Property 8. $\|\square\|_{\mathcal{U}_{0}^{h}}$ defined by $\|u\|_{\mathcal{U}_{0}^{h}}^{2}=b(u, u)+\gamma^{2}(u)$ is a norm over $\mathcal{U}_{0}^{h}$.

Proof. The only difficulty is to show that $\|u\|_{\mathcal{U}_{0}^{h}}^{2}=0$ leads to $u=0$ over $\Omega$. However, from

$$
0=b(u, u)=\sum_{E \in \mathbf{E}} \int_{\Omega_{E}} \eta k \operatorname{grad} u \cdot \operatorname{grad} \tilde{u} d \Omega+\int_{\partial_{2} \Omega}\left(\left(\mathbf{q}_{\mathbf{u}} \cdot \mathbf{n}\right)\left(\tilde{\mathbf{q}}_{\mathbf{u}} \cdot \mathbf{n}\right) / h+h k^{2} u \tilde{u}\right) d S
$$

it is clear that $u_{\mid \Omega_{E}}=a_{E}$ is constant over $\Omega_{E}$ and that $u=0$ over $\partial_{2} \Omega$.

It follows that $\gamma(u)$ is equal to

$$
\gamma(u)=\sup _{v \in \overline{\mathcal{U}}_{0}^{h}} \frac{1}{\left\|\mathbf{C}_{\mathbf{v}}\right\|_{L^{2}(\Omega)}} \operatorname{Re}\left(-i k\left(\sum_{E, E^{\prime} \in \mathbf{E}} \int_{\Gamma_{E E^{\prime}}} \mathbf{q}_{\mathbf{v}} \cdot \mathbf{n} a_{E^{\prime}} d S+\sum_{E \in \mathbf{E}} \int_{\Gamma_{E E \cap \partial \Omega}} \mathbf{q}_{\mathbf{v}} \cdot \mathbf{n} a_{E^{\prime}} d S\right)\right)=0 .
$$

Since condition (P) is satisfied in $\overline{\mathcal{U}}_{0}^{h}, u_{E}= \pm a, a$ being a constant over $\Omega$. From $u=0$ on $\partial_{2} \Omega$, one gets $u=0$ over $\Omega$.

Property 9. The solution $\bar{u} \in \mathcal{U}_{0}^{h}$ of $b(\bar{u}, v)=l(v) \forall v \in \mathcal{U}_{0}^{h}$ is such that

$$
\|\bar{u}\|_{\mathcal{U}_{0}^{h}} \leq\left(|l|_{\mathcal{U}_{0}^{h,}}^{2}+2\|l\|_{\mathcal{U}_{0}^{h,}}^{2}\right)^{1 / 2}
$$

Proof. Since $b(\bar{u}, v)=l(v) \forall v \in \mathcal{U}_{0}^{h}, b(\bar{u}, \bar{u}) \leq|l|_{\mathcal{U}_{0}^{h,}}\|\| \bar{u} \|_{\mathcal{U}_{0}^{h}}$, where $\mathcal{U}_{0}^{h}$, is the dual space of $\mathcal{U}_{0}^{h} .\|l\|_{\mathcal{U}_{0}^{h}}$, is another norm defined by $\|l\|_{\mathcal{U}_{0}^{h,}}=\sup _{v \in \overline{\mathcal{U}}_{0}^{h}} l(v) /\left\|\mathbf{C}_{\mathbf{v}}\right\|_{L^{2}(\Omega)}$ and $\gamma(\bar{u})=\sup _{v \in \overline{\mathcal{U}}_{0}^{h}} b(\bar{u}, v) /\left\|\mathbf{C}_{\mathbf{v}}\right\|_{L^{2}(\Omega)}=\sup _{v \in \overline{\mathcal{U}}_{0}^{h} l(v) /}$ $\left\|\mathbf{C}_{\mathbf{v}}\right\|_{L^{2}(\Omega)}=\|l\|_{\overline{\mathcal{U}}_{0}^{h, .}}$ Finally, one has $b(\bar{u}, \bar{u})+\gamma^{2}(\bar{u}) \leq \frac{1}{2}|l|_{\mathcal{U}_{0}^{h}}^{2}+\frac{1}{2}\left(b(\bar{u}, \bar{u})+\gamma^{2}(\bar{u})\right)+\|l\|_{\overline{\mathcal{U}}_{0}^{h,}}^{2}$. Therefore, Property 9 holds. 


\section{Coupling of the FEM and the VTCR}

The weak Trefftz discontinuous Galerkin formulation (12) can be used to couple the FEM and the VTCR. Let us divide $\Omega$ into two parts $\Omega_{1}$ and $\Omega_{2}$ and use the VTCR in $\Omega_{1}$ and the FEM in $\Omega_{2}$ (see the rightmost part of Fig. 1). The corresponding sets of subdomains are $\mathbf{E}_{1}$ and $\mathbf{E}_{2}$. In the working space $\mathcal{U}^{h}=\mathcal{U}_{1}^{h} \otimes \mathcal{U}_{2}^{h}$, for $E \in \mathbf{E}_{\mathbf{1}}$,

$$
\mathcal{U}_{E}^{h}=\left\{u^{h} \mid u^{h} \in \mathcal{V}_{E}^{h} ;(1+i \eta) \Delta u^{h}+k^{2} u^{h}+r_{d}=0 \text { on } \Omega_{E}\right\}
$$

and for $E \in \mathbf{E}_{\mathbf{2}}$,

$$
\mathcal{U}_{E}^{h}=\left\{u^{h} \mid u^{h} \in \mathcal{V}_{E}^{\prime h} ; \int_{\Omega_{E}}\left((1+i \eta) \Delta u^{h}+k^{2} u^{h}+r_{d}\right) \tilde{v}^{h} d \Omega=0 \forall v^{h} \in \mathcal{V}_{E, 0}^{\prime h}\right\} .
$$

Property 10. The coupled FEM/VTCR problem defined by the weak Trefftz discontinuous Galerkin formulation (12) has a unique solution if the number of internal modes of $\Omega_{2}$ is nonzero. Besides, $\|\square\|_{\mathcal{U}_{0}^{h}}=(b(\square, \square))^{1 / 2}$ is a norm over $\mathcal{U}_{0}^{h}$.

Proof. This property is a consequence of Properties 4 and 5.

Of course, the coupling between the FEM and the VTCR can still be used with Formulation (12) if the wave number $k$ is not the same in the two subdomains. The previous properties are still valid. $k$ has only to be replaced by its corresponding value in $\Omega_{1}$ and in $\Omega_{2}$. This will be illustrated by the example of the next section.

\section{Numerical illustrations}

In this section, the weak Trefftz discontinuous Galerkin formulation described in Section 3 is tested using two benchmark problems:

- The first problem is defined over a square domain divided into two subdomains with different wave numbers so that an incident wave gives rise to a reflected wave and a transmitted wave. This problem has an analytical solution, which is used to define the prescribed boundary conditions and to assess the quality of the approximate solution.

- The second problem is defined over a curved $L$-shaped domain with prescribed boundary conditions and different characteristics in the subdomains. In both cases, the weak Trefftz discontinuous Galerkin solution is compared to a FEM solution.

\subsection{The incident wave problem}

\subsubsection{Problem description}

The domain being considered (see Fig. 2) is a square $\Omega=[-0.5 \mathrm{~m} ; 0.5 \mathrm{~m}] \times[-0.5 \mathrm{~m} ; 0.5 \mathrm{~m}]$ divided into two subdomains: $\Omega_{a}=[-0.5 \mathrm{~m} ; 0.5 \mathrm{~m}] \times[-0.5 \mathrm{~m} ; 0 \mathrm{~m}]$ with $k_{a}=6.536 \mathrm{~m}^{-1}$ and $\eta_{a}=0.001$, and $\Omega_{b}=[-0.5 \mathrm{~m} ; 0.5 \mathrm{~m}] \times[0 \mathrm{~m} ; 0.5 \mathrm{~m}]$ with $k_{b}=29.412 \mathrm{~m}^{-1}$ and $\eta_{b}=0.001$. We focused on the problem with a homogeneous governing equation, so $r_{d}$ equals zero. The Robin boundary condition (the last equation of (1)) was applied along all the boundaries of $\Omega_{a}$ and $\Omega_{b}$ with $h=1$ and $g_{d}$ such that the exact solution is $u_{b}^{\text {ex }}=u_{\text {inc }}+u_{r}$ in $\Omega_{b}$ and $u_{a}^{\text {ex }}=u_{t}$ in $\Omega_{a}$, defining the solution in terms of a classical incident wave and giving rise to a reflected wave and a transmitted wave according to Descartes' laws. Such a solution with an incident wave of unit amplitude propagating at a $9^{\circ}$ angle from the vertical can be seen on the right-hand side of Fig. 2. This incident angle was chosen to be less than the critical angle so that the transmitted wave would propagate through $\Omega_{a}$.

$k_{b}$ was chosen to be greater than $k_{a}$, which can be seen in the solution shown in Fig. 2 in which the wavelength in $\Omega_{b}$ is clearly smaller than the wavelength in $\Omega_{a}$. Consequently, in order to test the coupling between the VTCR and the FEM, the solution in $\Omega_{b}$ (with the smaller wavelength) was described using the VTCR and the solution in $\Omega_{a}$ (with the larger wavelength) using the FEM.

The FEM model was defined using $Q 1$ discretization over the whole domain $\Omega_{a}$. The mesh was made of square elements regularly spaced along the $x$ and $y$ axes. Different mesh sizes $(10 \times 5$ elements, $20 \times 10$ elements and $40 \times 20$ elements) were tested in order to compare the meshes of increasing refinement in the FEM discretization. 

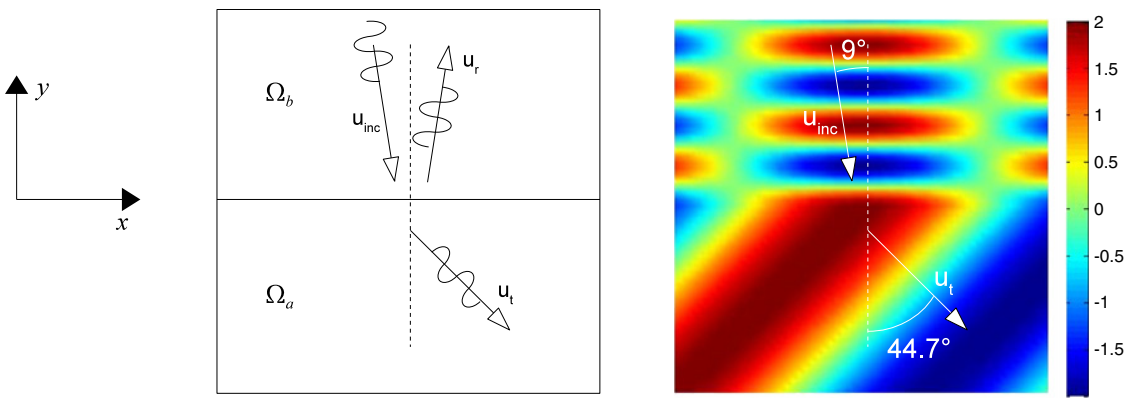

Fig. 2. Left: definition of the computational domain $\Omega$ for the example considered in Section 5.1. Right: the exact solution (plot of the real part) for the selected values of the physical data in $\Omega_{a}$ and $\Omega_{b}$.
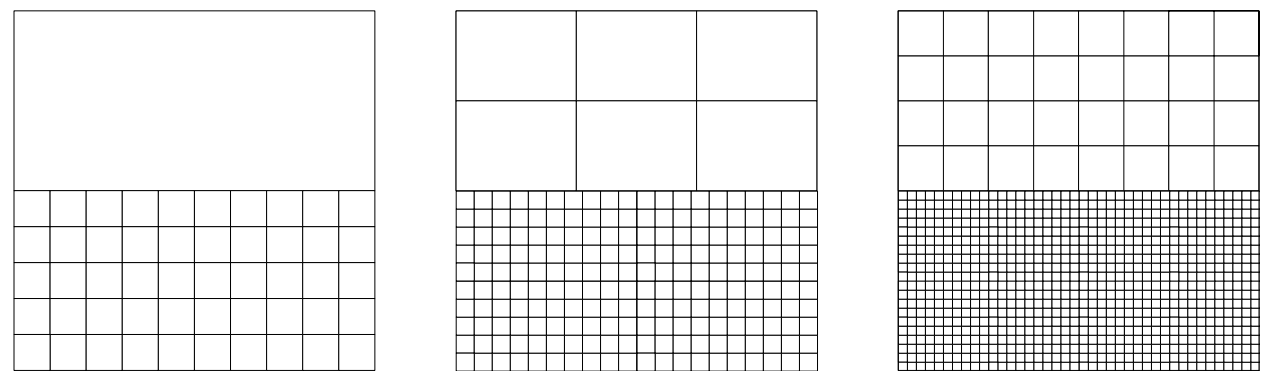

Fig. 3. Examples of meshes used to calculate the solution of the problem of Section 5.1. On the left, $\Omega_{b}$ contains only one $\Omega_{E}$ subdomain and $\Omega_{a}$ is viewed as a single $\Omega_{E}$ subdomain discretized using $10 \times 5$ elements. In the middle, $\Omega_{b}$ contains $3 \times 2 \Omega_{E}$ subdomains and $\Omega_{a}$ is viewed as a single $\Omega_{E}$ subdomain discretized using $20 \times 10$ elements. On the right, $\Omega_{b}$ contains $8 \times 4 \Omega_{E}$ subdomains and $\Omega_{a}$ is viewed as a single $\Omega_{E}$ subdomain discretized with $40 \times 20$ elements.

These meshes, shown in Fig. 3, correspond respectively to about 10, 20 and 40 elements per wavelength. For the VTCR, in order to compare the results obtained with increasing numbers of VTCR subdomains, we discretized $\Omega_{b}$ into $1,3 \times 2$ and $8 \times 4$ subdomains $\Omega_{E}$ as shown in Fig. 3. It should be noticed that the FEM and the VTCR meshes did not always match along the common boundary of $\Omega_{a}$ and $\Omega_{b}$.

As explained in Section 2.2, the VTCR approximation uses solutions $u^{h} \in \mathcal{U}^{h}$ which are exact solutions of the Helmholtz equation. Many different choices can be made concerning these exact solutions (see [29] for a global overview). Here, because of the ease with which this could be implemented, we described $\mathcal{U}^{h}$ using regularly spaced rays propagating in all directions in $\left[0 ; 2 \pi\right.$ [. Therefore, in each subdomain $\Omega_{E}$ of $\Omega_{b}$, the approximate solution $u^{h}$ was generated as a sum of $N_{E}$ propagative waves $e^{i \mathbf{k}_{\mathbf{E}, \mathbf{j}} \cdot\left(\mathbf{x}-\mathbf{x}_{\mathbf{E}}\right)}$, where $\mathbf{k}_{\mathbf{E}, \mathbf{j}}$ is the wave vector of amplitude $k_{b} / \sqrt{1+i \eta_{b}}$ along direction $\theta_{j}=2 \pi j / N_{E}$ for $j=0 \ldots N_{E}-1$, and $\mathbf{x}_{\mathbf{E}}$ is the geometrical center of $\Omega_{E} . N_{E}$ corresponds to the number of waves used in each VTCR subdomain $\Omega_{E}$. With today's level of development of the VTCR, the number of regularly oriented waves which must be used in the VTCR subdomain $\Omega_{E}$ to achieve a good numerical solution is known a priori and is equal to $N_{E}=\left[2 \cdot k \cdot \operatorname{diam}\left(\Omega_{E}\right)\right]$, where [ $\left.\square\right]$ denotes the integer part, $k$ is the wave number and $\operatorname{diam}\left(\Omega_{E}\right)$ is the diameter of $\Omega_{E}$ (see [28] for more details). Here, we selected $N_{E}=10,20,30,40$ and 60, even though these do not satisfy this criterion, because we wanted to find out how the weak Trefftz discontinuous Galerkin solution behaves as a function of the VTCR refinement.

\subsubsection{Study of the weak Trefftz discontinuous Galerkin solution as a function of the FEM refinement}

Fig. 4 shows a comparison of the results obtained using the weak Trefftz discontinuous Galerkin approach in which $\Omega_{a}$ was discretized into one $\Omega_{E}$ subdomain with $10 \times 5,20 \times 10$ and $40 \times 20$ elements, and $\Omega_{b}$ was discretized using $3 \times 2 \Omega_{E}$ subdomains with $N_{E}=30$ rays. This comparison was chosen because this VTCR discretization is refined enough to ensure a good enough solution in $\Omega_{b}$ and only the quality of the solution in $\Omega_{a}$ needs to be considered. (See the heuristic geometric criterion in [28] which leads to an appropriate number of rays to be used in a VTCR subdomain.) It can be observed that the solution converges toward the exact solution of Fig. 2 when the number of FEM elements becomes sufficiently large. For the $10 \times 5$ FEM mesh, the solution in $\Omega_{a}$ was unsatisfactory and seemed 

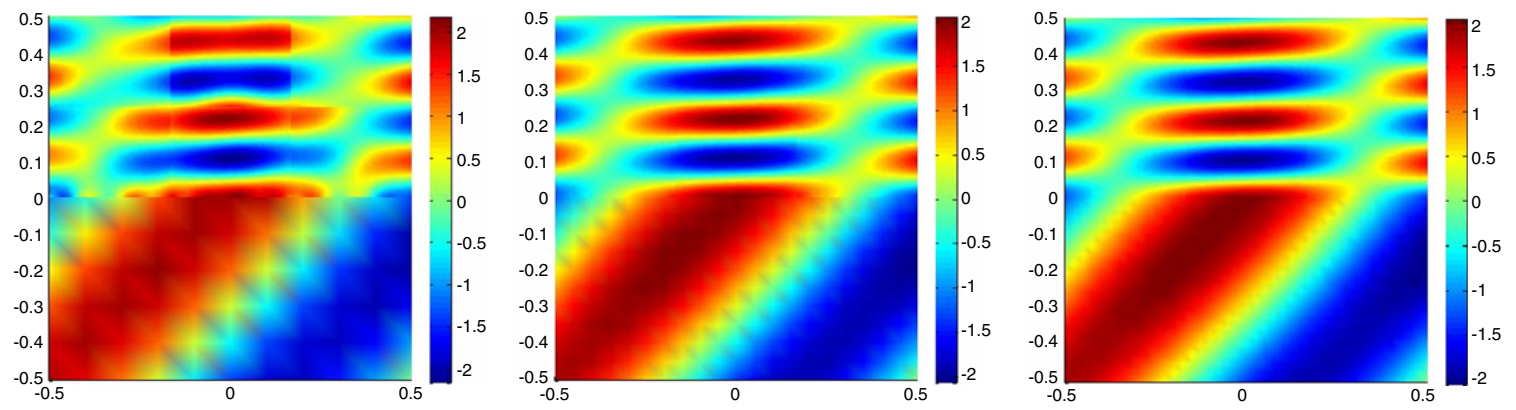

Fig. 4. The solution obtained using the weak Trefftz discontinuous Galerkin method for the example of Section 5.1 . $\Omega_{b}$ was discretized using $3 \times 2$ $\Omega_{E}$ subdomains with $N_{E}=30$ rays. $\Omega_{a}$ was discretized into a single $\Omega_{E}$ subdomain using $10 \times 5$ (left), $20 \times 10$ (middle) and $40 \times 20$ (right) elements respectively.
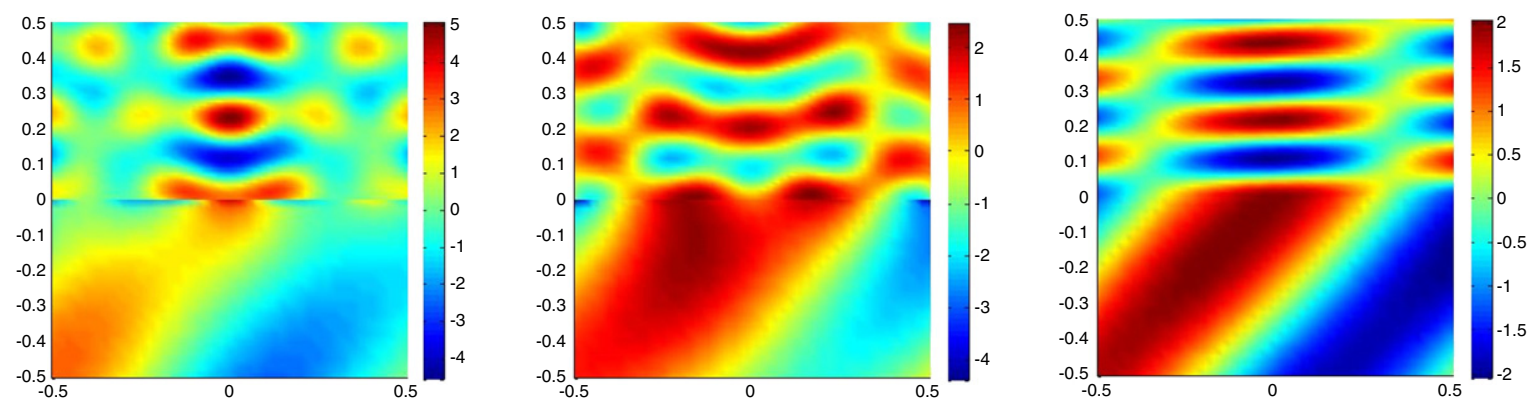

Fig. 5. The solution obtained using the weak Trefftz discontinuous Galerkin method for the example of Section 5.1. $\Omega_{b}$ was discretized using a single $\Omega_{E}$ subdomain with $N_{E}=10$ (left), 30 (middle) and 60 (right) rays. $\Omega_{a}$ was discretized into a single $\Omega_{E}$ subdomain with $40 \times 20$ elements.

to perturb the solution in $\Omega_{b}$. As soon as the FEM became more refined, the solution corresponded increasingly to the exact solution.

\subsubsection{Study of the weak Trefftz discontinuous Galerkin solution as a function of the VTCR refinement}

Fig. 5 shows a comparison of the results obtained using the weak Trefftz discontinuous Galerkin approach in which $\Omega_{a}$ was discretized into a single $\Omega_{E}$ subdomain with $40 \times 20$ elements and $\Omega_{b}$ was discretized into a single $\Omega_{E}$ subdomain with $N_{E}=10,30$ and 60 rays. Contrary to $N_{E}=60, N_{E}=10$ and $N_{E}=30$ were insufficiently large numbers of rays to ensure a good enough quality of the result in the VTCR domain according to the heuristic criterion in [28]. Consequently, as can be seen in Fig. 5, even though the discretization of the FEM domain was refined enough (as shown by the results in Fig. 4), the global solutions were unsatisfactory. However, when a sufficiently large number of rays was used in the VTCR domain (60 in this case), the solution matched the exact solution.

A similar conclusion can be reached for the comparison in Fig. 6, where $\Omega_{a}$ was discretized into a single $\Omega_{E}$ subdomain with $40 \times 20$ elements and $\Omega_{b}$ was discretized using $3 \times 2 \Omega_{E}$ subdomains with $N_{E}=20,30$ and 40 rays each. The FEM discretization was sufficiently refined (based on the results of Fig. 4), and the number of VTCR rays was sufficient for $N_{E}=30$ and $N_{E}=40$ to lead to good results, but that was not the case for $N_{E}=20$. Consequently, the solution with $N_{E}=20$ was unsatisfactory, whereas the solutions with $N_{E}=30$ and $N_{E}=40$ were good.

Finally, Fig. 7 shows a comparison of several calculations in which $\Omega_{a}$ was discretized into a single $\Omega_{E}$ subdomain with $40 \times 20$ elements and $\Omega_{b}$ was discretized using $8 \times 4 \Omega_{E}$ subdomains with $N_{E}=10,20$ and 60 rays each. As mentioned before, the FEM discretization was refined enough. $N_{E}=20$ was a large enough number of VTCR rays for a good VTCR discretization, which was not the case of $N_{E}=10$. Consequently, the solution with $N_{E}=20$ is reliable, but the solution with $N_{E}=10$ is not. Regarding the solution obtained with $N_{E}=60$, some small discontinuities can be observed at the boundaries of the VTCR subdomains. This was the result of using too many rays in the VTCR discretization, making the conditioning number of the matrix of the weak Trefftz discontinuous Galerkin formulation very large, thus degrading the quality of the solution. Preconditioners or adapted iterative solvers could be used to 

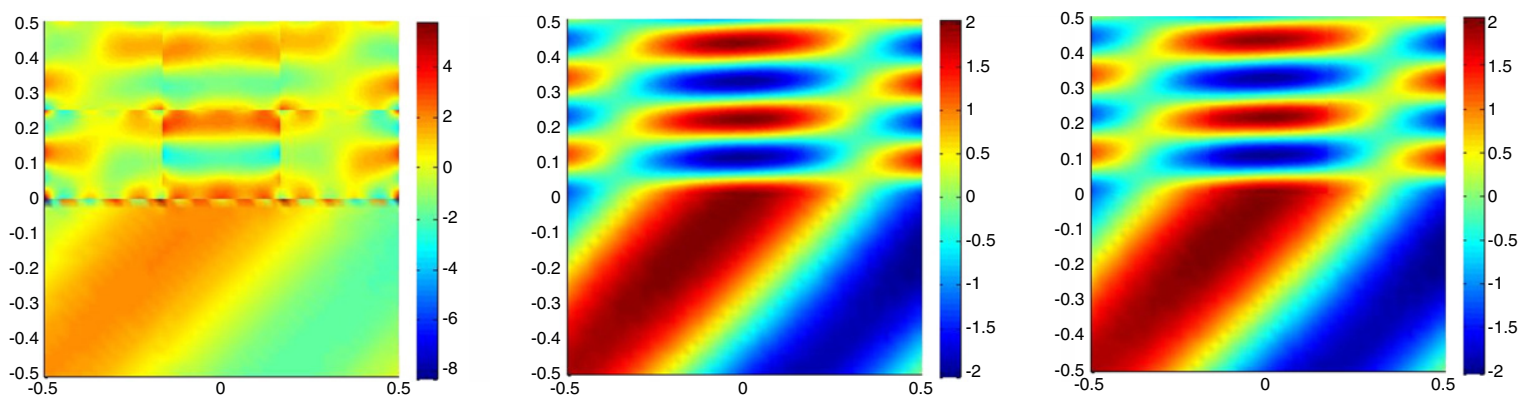

Fig. 6. The solution obtained using the weak Trefftz discontinuous Galerkin method for the example of Section 5.1. $\Omega_{b}$ was discretized using $3 \times 2$ $\Omega_{E}$ subdomains with $N_{E}=20$ (left), 30 (middle) and 40 rays (right). $\Omega_{a}$ was discretized into a single $\Omega_{E}$ subdomain with $40 \times 20$ elements.
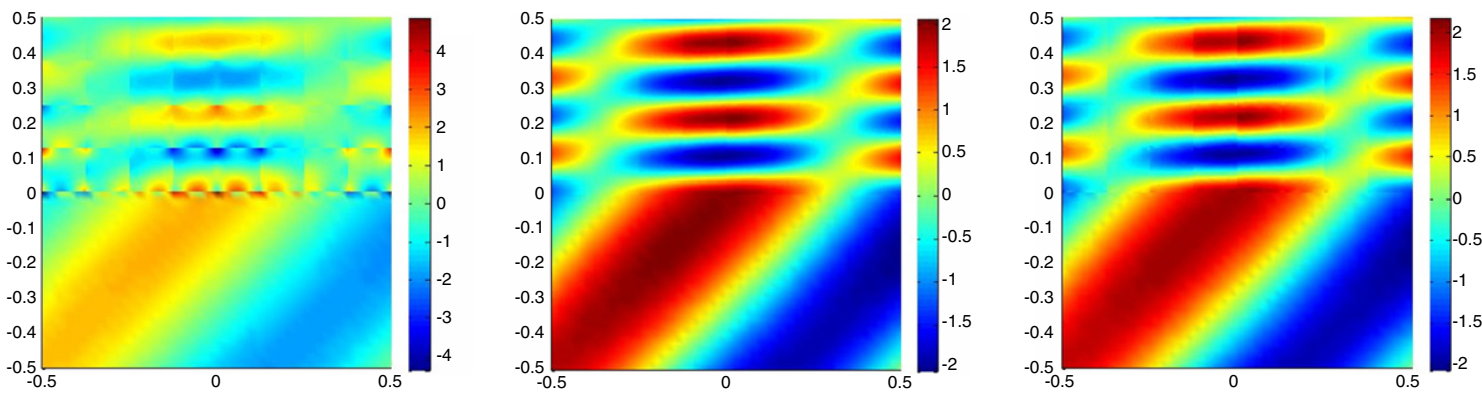

Fig. 7. The solution obtained using the weak Trefftz discontinuous Galerkin method for the example of Section 5.1. $\Omega_{b}$ was discretized using $8 \times 4$ $\Omega_{E}$ subdomains with $N_{E}=10$ (left), 20 (middle) and 60 rays (right). $\Omega_{a}$ was discretized into a single $\Omega_{E}$ subdomain with $40 \times 20$ elements.

alleviate this problem. This was not done in this paper in order to achieve a fair conclusion concerning the new formulation (12).

\subsubsection{Study of the numerical properties}

Now, let us examine how the weak Trefftz discontinuous Galerkin solution of the problem is affected by the coupling between the FEM and the VTCR. In order to do that, let us compare the solutions given by the FEM description applied to both $\Omega_{a}$ and $\Omega_{b}$ using a regular mesh, the VTCR description applied to both $\Omega_{a}$ and $\Omega_{b}$, and a hybrid description in which $\Omega_{a}$ is discretized using a regular FEM mesh and $\Omega_{b}$ is discretized using the VTCR with a single subdomain $\Omega_{E}$ following the numerical strategy presented in this paper. To measure the quality of each solution, we use the relative $L^{2}$ error $\left\|u^{\mathrm{ex}}-u^{h}\right\| /\left\|u^{\mathrm{ex}}\right\|=\sqrt{\int_{\Omega}\left\|u^{\mathrm{ex}}-u^{h}\right\|^{2} d \Omega / \int_{\Omega}\left\|u^{\mathrm{ex}}\right\|^{2} d \Omega}$. The convergence curves are shown in Fig. 8.

A comparison of the curve corresponding to the FEM solution (which decreases with the usual convergence rate) and the curve corresponding to the VTCR solution shows how advantageous it is to use a wave description to model a vibration problem. This has already been observed in the previous papers on the VTCR (see for example [27]). A comparison of these two curves to the 'VTCR(60)/FEM' curves (corresponding to 60 rays being used in the VTCR subdomain $\Omega_{b}$, which, according to the evidence given above, is sufficient to ensure that the VTCR has converged) shows that coupling the VTCR and the FEM can be worthwhile. Indeed, the use of this hybrid description led to an error which was smaller by more than an order of magnitude than that of a pure FEM calculation. However, in this example, the hybrid description was less efficient than a pure VTCR description. However, it should be recalled that our primary objective with the weak Trefftz discontinuous Galerkin description (which enables one to couple two different descriptions) is flexibility rather than the most efficient calculation in all cases. In Fig. 8, we also plotted the 'VTCR(20)/FEM' curve, which corresponds to only 20 rays being used in the VTCR domain. According to the evidence given above, this number of rays in the VTCR description is too small to ensure the convergence of the solution in subdomain $\Omega_{b}$. It is clear that the error remained high and, thus, the approximate solution remained poor, even when the number of degrees of freedom in the FEM domain was increased. This is to be expected not unusual because such a VTCR solution is not refined enough to converge in all cases. 


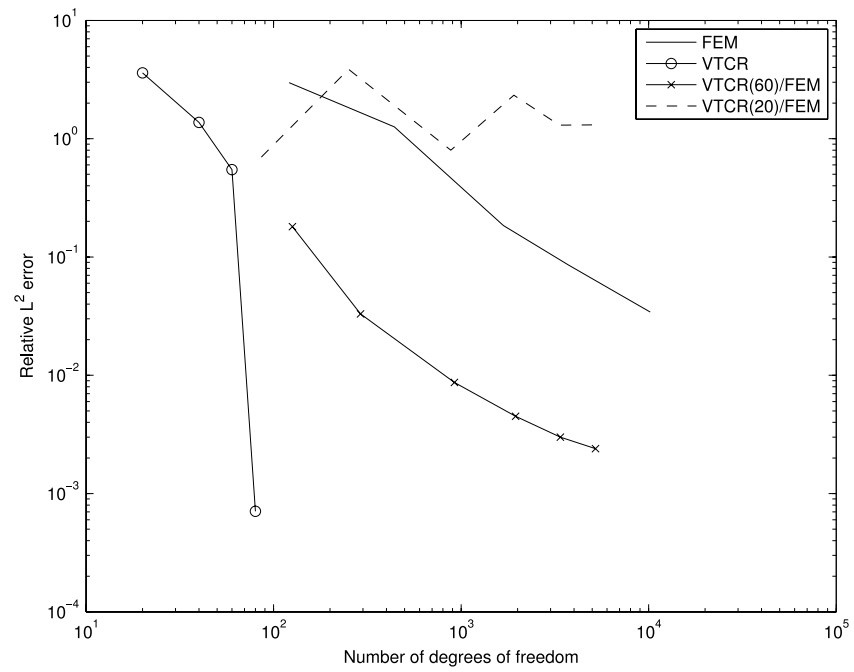

Fig. 8. The convergence curves for the example of Section 5.1 using various types of descriptions. The 'FEM' curve corresponds to the solution obtained with a pure FEM description of both $\Omega_{a}$ and $\Omega_{b}$ using a regular mesh. The 'VTCR' curve corresponds to the solution obtained with a pure VTCR description of both $\Omega_{a}$ and $\Omega_{b}$ using the same number of degrees of freedom in both subdomains. The 'VTCR(X)/FEM' curves correspond to the solution obtained with a FEM description of $\Omega_{a}$ using a regular mesh and a VTCR description of $\Omega_{b}$ using 'X' rays.

Table 1

The condition number of the global system matrix of the example of Section 5.1.4. $h$ denotes the size of the FEM mesh used in $\Omega_{a}$ and 'VTCR DOFs' corresponds to the number of rays used in $\Omega_{b}$.

\begin{tabular}{lllll}
\hline VTCR DOFs & $1 / h=20$ & $1 / h=40$ & $1 / h=60$ & $1 / h=80$ \\
\hline 20 & $1.3 \times 10^{4}$ & $1.3 \times 10^{4}$ & $3.3 \times 10^{4}$ & $5.2 \times 10^{4}$ \\
40 & $1.0 \times 10^{4}$ & $1.9 \times 10^{4}$ & $4.2 \times 10^{4}$ & $8.1 \times 10^{4}$ \\
50 & $1.9 \times 10^{8}$ & $9.0 \times 10^{9}$ & $8.0 \times 10^{8}$ & $1.1 \times 10^{9}$ \\
60 & $4.8 \times 10^{15}$ & $3.8 \times 10^{15}$ & $4.7 \times 10^{14}$ & $7.7 \times 10^{14}$ \\
\hline
\end{tabular}

Furthermore, it is well-known that the Trefftz methods such as the VTCR lead to ill-conditioned global system matrices. Table 1 shows a comparison of the conditioning numbers of the global system resulting from the weak Trefftz discontinuous Galerkin formulation with various model refinements, using the FEM in $\Omega_{a}$ and the VTCR with a single subdomain in $\Omega_{b}$. It can be observed that the conditioning number is greatly affected by the number of rays used in the VTCR, whereas the refinement of the FEM mesh has little influence.

\subsection{The L-shape problem}

\subsubsection{Problem description}

Turning now to focus on the homogeneous problem of a curved $L$-shape domain whose dimensions and boundary conditions are defined in Fig. 9, we consider two cases. In Case 1, the whole domain is filled with a single fluid with a wave number $k_{a}=6.5 \mathrm{~m}^{-1}$ and a damping coefficient $\eta_{a}=0.001$. In Case 2, the domain contains two fluids with different wave numbers $k_{a}=6.536 \mathrm{~m}^{-1}$ and $k_{b}=29.412 \mathrm{~m}^{-1}$ and the same damping coefficient $\eta_{a}=\eta_{b}=0.001$. In Case 2, as shown in Fig. 9, the fluid with the smaller wave number lies in the $0.4 \mathrm{~m} \times 2 \mathrm{~m}$ rectangular bottom right part of the domain. The boundary conditions are Robin conditions (the last equation of (1)) with $h=0.001$ and $g_{d}=0$ or $1 \mathrm{~m}^{-1}$ (see Fig. 9).

Since there is no known exact solution for this example, a reference solution was calculated using finite element analysis. In order to do that, $Q 1$ discretization with a sufficiently refined regular mesh was used to ensure at least 30 elements per wavelength, even in the worst case where $\Omega$ is filled with two fluids, which alleviates the pollution effects almost entirely. This solution was considered to be refined enough to be reliable. 

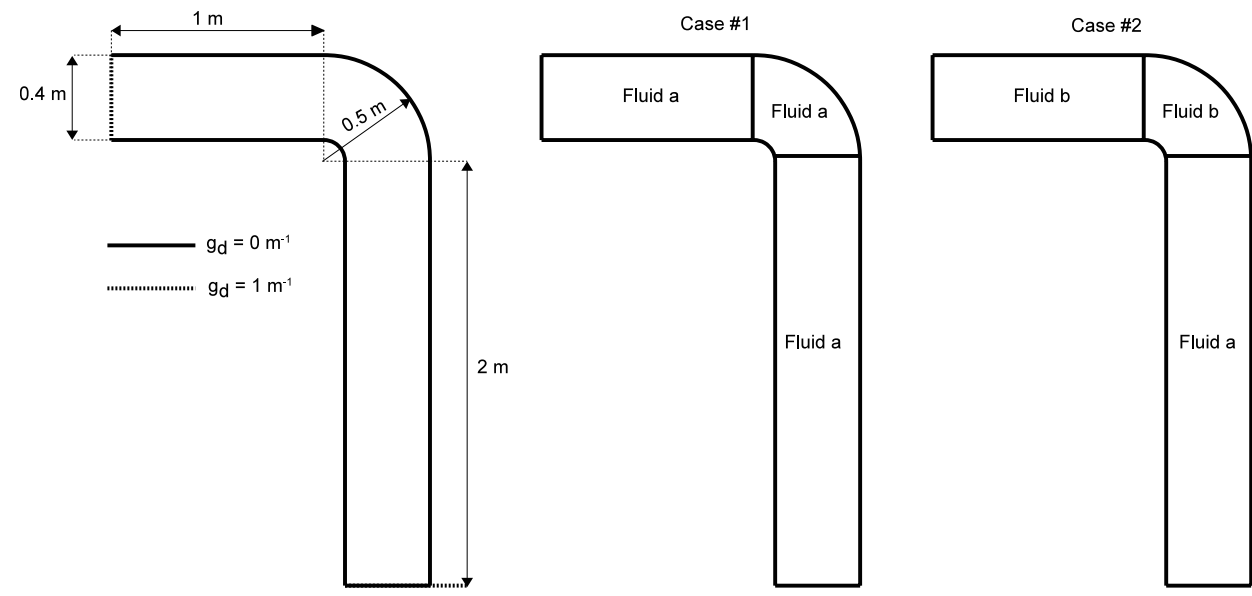

Fig. 9. Left: definition of the computational domain $\Omega$ and boundary conditions for the example considered in Section 5.2. Middle: characteristics of the fluid for Case 1. Right: characteristics of the fluid for Case 2. $k_{a}=6.536 \mathrm{~m}^{-1}, \eta_{a}=0.001, k_{b}=29.412 \mathrm{~m}^{-1}, \eta_{b}=0.001$.
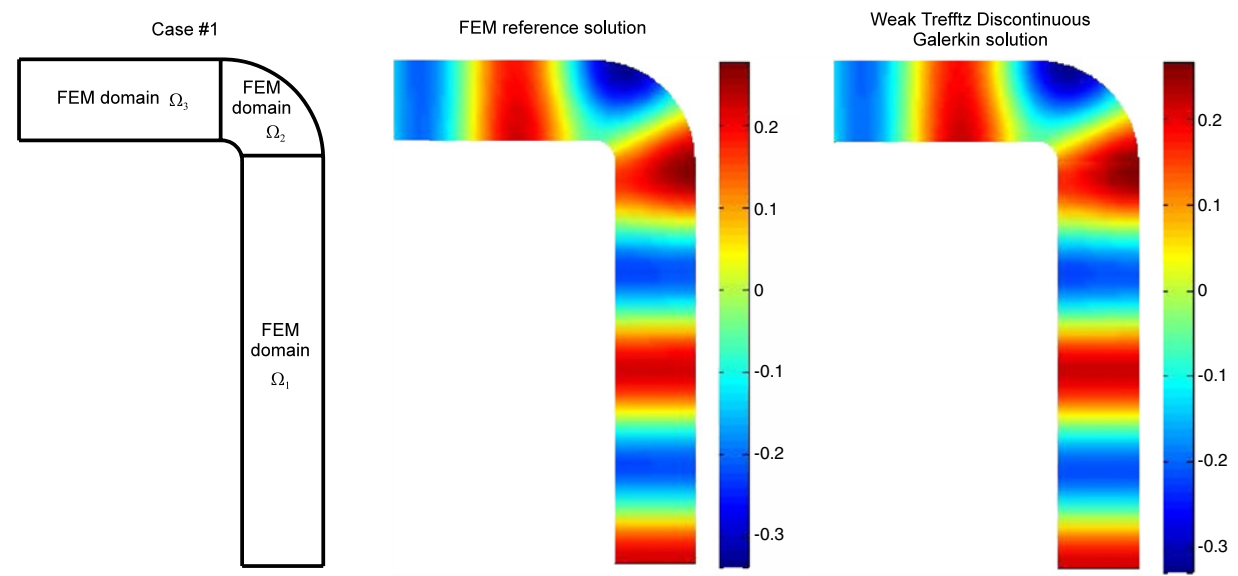

Fig. 10. Left: the selected subdomains and meshes for Case 1 of Fig. 9. Middle: the FEM reference solution. Right: the numerical results obtained with the weak Trefftz discontinuous Galerkin formulation.

\subsubsection{FEM-to-FEM coupling}

Let us consider Case 1 in which $\Omega$ is filled with only one fluid (the middle case of Fig. 9). $\Omega$ was discretized with 3 subdomains as shown in Fig. 10: $\Omega_{1}$ (the $0.4 \mathrm{~m} \times 2 \mathrm{~m}$ rectangular bottom right part), $\Omega_{2}$ (the curved upper right part) and $\Omega_{3}$ (the $1 \mathrm{~m} \times 0.4 \mathrm{~m}$ rectangular upper left part). In $\Omega_{1}$, we used a regular mesh with 10 elements along the $x$ axis and 40 elements along the $y$ axis, (451 DOFs). In $\Omega_{2}$, we used a regular mesh with 9 elements along the radial axis and 16 elements along the circumferential axis (170 DOFs). In $\Omega_{3}$, we used a regular mesh with 20 elements along the $x$ axis and 8 elements along the $y$ axis (189 DOFs). These meshes were chosen in order to have at least 20 elements per wavelength and, thus, avoid pollution effects. In addition, due to the number of elements chosen, the meshes in $\Omega_{1}, \Omega_{2}$ and $\Omega_{3}$ were nonconforming. The numerical result is shown on the right of Fig. 10. By comparing this solution with the reference solution in the center of Fig. 10, it can be observed that the proposed approach works perfectly. Indeed, the method was capable of recovering the reference solution even though the unknown $u^{h}$ was discontinuous across the model. This example illustrates the benefit of such an approach which can provide an accurate FEM result even with models whose meshes are nonconforming.

\subsubsection{VTCR-to-FEM coupling}

Now let us consider Case 2 in which $\Omega$ is filled with two fluids (on the right of Fig. 9). We used the mesh shown on the left of Fig. 11, combining the FEM and the VTCR strategies. $\Omega_{1}$, where the wave number is smaller, was modeled using the FEM with 10 elements along the $x$ axis and 40 elements along the $y$ axis (451 DOFs). The VTCR 

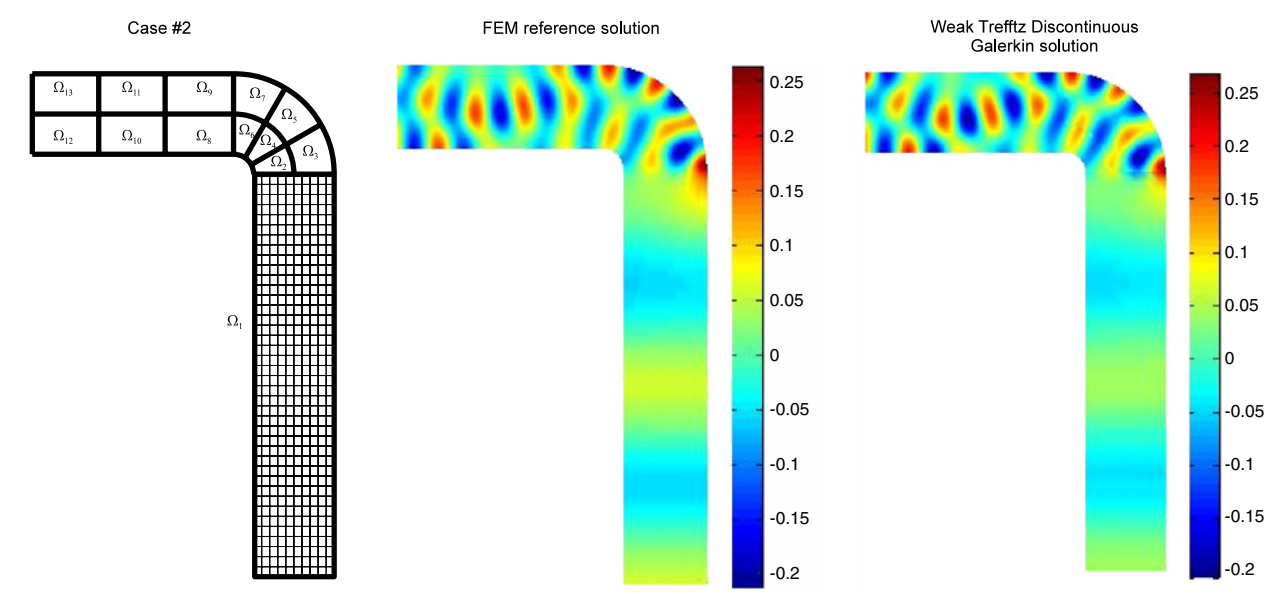

Fig. 11. Left: the selected subdomains and meshes for Case 2 of Fig. 9. Middle: the FEM reference solution. Right: the numerical result obtained with the weak Trefftz discontinuous Galerkin formulation.

approximation was used in subdomains $\Omega_{E}, E \in\{2 \ldots 13\}$. In all these subdomains, the number $N_{E}$ of regularly oriented rays satisfied $N_{E}=\left[2 . k \cdot \operatorname{diam}\left(\Omega_{E}\right)\right]$, where [ $\left.\square\right]$ denotes the integer part, $k$ the wave number and $\operatorname{diam}\left(\Omega_{E}\right)$ the diameter of $\Omega_{E}$. This choice ensured that the VTCR subdomains had enough DOFs to achieve a reliable solution (see the heuristic criterion in [28]). The result is shown in Fig. 11. One can see that, overall, the solution is similar to the FEM reference solution. Indeed, almost all the vibration peaks are located in the right place and have the right amplitude. Thus, this example validates the proposed approach and shows that the weak Trefftz discontinuous Galerkin formulation (12) is capable of solving this relatively complex numerical example which mixes different types of approximations with different physics. However, in the result of Fig. 11 some small discontinuities between the VTCR and the FEM domains can be detected at the common boundary. These discontinuities are due to the FEM mesh selected, which is not refined enough near the common boundary. Indeed, near that boundary, local vibration phenomena appear in $\Omega_{1}$ with wavelengths which are very close to that of fluid $b$. However, since these effects correspond to evanescent waves, they are localized at the boundary. Therefore, in order to get a better solution with almost no visible discontinuity, the FEM mesh should be refined near this boundary alone.

\section{Conclusion}

In the VTCR proposed in [17] for the calculation of medium-frequency phenomena, the solution of a vibrational problem is sought in an approximation space which is spanned by the exact solutions of the governing equation in the form of propagative and evanescent waves. These shape functions are discontinuous. The transmission conditions at the boundaries of the substructures are satisfied, thanks to a dedicated variational formulation which can be viewed as the Trefftz version of a discontinuous Galerkin formulation.

Section 2 of this paper identified strong links between the VTCR and the discontinuous Galerkin methods and provided some basic mathematical results in the case of the Helmholtz problem, showing, in particular, that the discrete problem makes sense. An extension to vibro-acoustic problems should present no difficulty.

We also introduced a new formulation for the resolution of vibration problems, called the weak Trefftz discontinuous Galerkin formulation, in which the Trefftz constraint (the need to satisfy the governing equation) is weakened. Section 3 provided some basic mathematical background for this formulation which, again, shows that the discrete problem makes sense. Moreover, it was shown that different numerical models (namely the VTCR and the FEM) can be easily coupled and some numerical illustrations were presented.

This weak Trefftz discontinuous Galerkin formulation seems to be a promising computational method which can foster new approaches to the resolution of engineering problems. It can be easily extended to other problems, such as quasi-static problems. Its main interest is its ability to couple different types of numerical models. Another great advantage is that it does not require the use of homogeneous substructures. Furthermore, in the case where the substructures are elements, its flexibility enables elements to be removed or added easily. Finally, the application of the weak Trefftz discontinuous Galerkin formulation to 3D problems can be based on the 3D VTCR extension 
of [26], which considers a 3D acoustic car cavity in the medium-frequency range. An extension to vibro-acoustic problems would present no particular difficulty and will be addressed in future works.

\section{References}

[1] O.C. Zienkiewicz, The Finite Element Method, McGraw-Hill, 1977.

[2] E. Trefftz, Ein gegenstuck zum ritzschen verfahren, in: Second International Congress on Applied Mechanics, 1926, pp. 131-137.

[3] T. Strouboulis, R. Hidajat, Partition of unity method for Helmholtz equation: q-convergence for plane-wave and wave-band local bases, Appl. Math. 51 (2006) 181-204.

[4] O. Cessenat, B. Despres, Application of an ultra weak variational formulation of elliptic PDEs to the two-dimensional Helmholtz problem, SIAM J. Numer. Anal. 35 (1998) 255-299.

[5] T. Huttunen, J. Kaipio, P. Monk, An ultra-weak method for acoustic fluid-solid interaction, J. Comput. Appl. Math. 213 (2008) 166-185.

[6] C.J. Gittelson, R. Hiptmair, I. Perugia, Plane wave discontinuous Galerkin methods: analysis of the $h$-version, ESAIM Math. Model. Numer. Anal. 43 (2009) 297-331.

[7] R. Hiptmair, A. Moiola, I. Perugia, Plane wave discontinuous Galerkin methods for the 2D Helmholtz equation: analysis of the $p$-version, SIAM J. Numer. Anal. 49 (2011) 264-284.

[8] P. Monk, D. Wang, A least-squares method for the Helmholtz equation, Comput. Methods Appl. Mech. Engrg. 175 (1999) $121-136$.

[9] G. Gabard, P. Gamallo, T. Huttunen, A comparison of wave-based discontinuous Galerkin, ultra-weak and least-square methods for wave problems, Internat. J. Numer. Methods Engrg. 85 (2011) 380-402.

[10] C. Farhat, I. Harari, L. Franca, The discontinuous enrichment method, Comput. Methods Appl. Mech. Engrg. 190 (2001) $6455-6479$.

[11] C. Farhat, R. Tezaur, J. Toivanen, A domain decomposition method for discontinuous Galerkin discretizations of Helmholtz problems with plane waves and Lagrange multipliers, Internat. J. Numer. Methods Engrg. 78 (2009) 1513-1531.

[12] P. Bouillard, S. Suleau, Element-free Galerkin solutions for Helmholtz problems: formulation and numerical assessment of the pollution effect, Comput. Methods Appl. Mech. Engrg. 162 (1998) 317-335.

[13] E. Perrey-Debain, J. Trevelyan, P. Bettess, Wave boundary elements: a theoretical overview presenting applications in scattering of short waves, Eng. Anal. Bound. Elem. 28 (2004) 131-141.

[14] H. Bériot, E. Perrey-Debain, M. Ben Tahar, C. Vayssade, Plane wave basis in Galerkin BEM for bidimensional wave scattering, Eng. Anal. Bound. Elem. 34 (2010) 130-143.

[15] W. Desmet, P. Sas, D. Vandepitte, An indirect Trefftz method for the steady-state dynamic analysis of coupled vibro-acoustic systems, Comput. Assist. Mech. Eng. Sci. 8 (2001) 271-288.

[16] B. Van Genechten, O. Atak, B. Bergen, E. Deckers, S. Jonckheere, J.S. Lee, A. Maressa, K. Vergote, B. Pluymers, D. Vandepitte, et al., An efficient wave based method for solving Helmholtz problems in three-dimensional bounded domains, Eng. Anal. Bound. Elem. 36 (2012) 63-75.

[17] P. Ladevèze, A new computational approach for structure vibrations in the medium frequency range, C. R. Acad. Sci. Paris 332 (1996) 849-856.

[18] P. Ladevèze, M. Chevreuil, A new computational method for transient dynamics including the low-and the medium-frequency ranges, Internat. J. Numer. Methods Engrg. 64 (2005) 503-527.

[19] P. Ladevèze, L. Arnaud, P. Rouch, C. Blanzé, The variational theory of complex rays for the calculation of medium-frequency vibrations, Eng. Comput. 18 (2001) 193-214.

[20] P. Ladevèze, A. Barbarulo, H. Riou, L. Kovalevsky, MID-FREQUENCY - CAE Methodologies for Mid-Frequency Analysis in Vibration and Acoustics, Leuven University Press, 2012, pp. 155-203. (Chapter 5) LMT Cachan.

[21] C. Hochard, P. Ladevèze, L. Proslier, A simplified analysis of elastic structures, Eur. J. Mech. A Solids 12 (1993) $509-535$.

[22] B. Cockburn, G.E. Karniadakis, C.-W. Shu, Discontinuous Galerkin Methods: Theory, Computation, and Applications, in: Lecture Notes in Computational Science and Engineering, vol. 11, 2000.

[23] D.N. Arnold, F. Brezzi, B. Cockburn, L.D. Marini, Unified analysis of discontinuous Galerkin methods for elliptic problems, SIAM J. Numer. Anal. 39 (2002) 1749-1779.

[24] C.E. Baumann, J.T. Oden, A discontinuous hp finite element method for convection diffusion problems, Comput. Methods Appl. Mech. Engrg. 175 (1999) 311-341.

[25] S. Prudhomme, F. Pascal, J.T. Oden, A. Romkes, A priori error estimate for the Baumann-Oden version of the discontinuous Galerkin method, C. R. Acad. Sci. Paris Sér. I Math. 332 (2001) 851-856.

[26] L. Kovalevsky, P. Ladevèze, H. Riou, M. Bonnet, The variational theory of complex rays for three-dimensional Helmholtz problems, J. Comput. Acoust. (2012).

[27] H. Riou, P. Ladevèze, B. Sourcis, The multiscale VTCR approach applied to acoustics problems, J. Comput. Acoust. 16 (2008) $487-505$.

[28] H. Riou, P. Ladevèze, B. Sourcis, B. Faverjon, L. Kovalesvky, An adaptative numerical strategy for the medium-frequency analysis of Helmholtz's problem, J. Comput. Acoust. 20 (2012) 1250001.

[29] L. Kovalevsky, P. Ladevèze, H. Riou, The Fourier version of the variational theory of complex rays for medium-frequency acoustics, Comput. Methods Appl. Mech. Engrg. 225 (2012) 142-153. 